\title{
Re: Multicenter External Validation and Comparison of Stone Scoring Systems in Predicting Outcomes after Percutaneous Nephrolithotomy
}

\author{
Tailly TO1,2, Okhunov Z33, Nadeau BR4, Huynh MJ1, Labadie K³, Akhavein A5, Violette PD1, \\ Olvera-Posada D1, Alenezi H¹, Amann J4, Bird VG55, Landman J3, Smith AD6, Denstedt JD1, Razvi H1
}

1 Western University Department of Surgery, Clinic of Urology, Ontario, Canada

2 Ghent University Hospital, Clinic of Urology, Ghent, Belgium

3 University of California, Department of Urology, Irvine, California

4 Western University Faculty of Medicine, Department of Radiology, Ontrario, Canada,

5 University of Florida, Department of Urology, Gainesville, Florida, USA

6North Shore LIJ Health System, The Smith Institute for Urology, New York, USA

J Endourol 2016;30:594-601. doi: 10.1089/end.2015.0700. Epub 2016 Feb 5.

\section{EDITORIAL COMMENT}

There was no stone scoring system for preoperative patient counseling and standardization until Guy's stone score was developed in 2011.S.T.O.N.E Nephrolithometry and Clinical Research Office of Endourological Society nomogram have been developed, respectively. The present study is the largest multicenter cohort study including 586 patients, retrospectively evaluating and comparing these three scoring systems at four academic institutions. The authors reported similar results regarding estimation of stone-free rates (SFR) with all the three scoring systems after a single session percutaneous nephrolitotomy (PCNL). They also noted that none of these scoring systems have significantly added predictive accuracy over stone size alone as a predictor of SFR. The patients were stratified into low-, intermediate-, high- and very high-risk groups for relative risk for residual stone for all scoring systems and it was seen that residual stone risk after PCNL surgery increases with increased risk group stratification. With regard to complications, none of the scoring systems strongly predicted complications. Only S.T.O.N.E score was found to be an independent predictor of operation time. As a result, all the three scoring systems are useful and equally effective in prediction of SFR despite specific limitations. Urological surgeons may benefit from these scoring systems for estimating case complexity and preoperative patient counseling. Further researches on scoring systems are mandatory especially for prediction of complications.

Ozan Bozkurt, MD

\section{Re: Chemokines in Cancer}

\section{Chow MT, Luster AD}

Harvard Medical School, Massachusetts General Hospital, Center for Immunology and Inflammatory Diseases, Division of Rheumatology, Allergy and Immunology, Boston, Massachusetts

Cancer Immunol Res 2014;2:1125-1131. doi: 10.1158/2326-6066.CIR-14-0160.

\section{EDITORIAL COMMENT}

Chemokines are chemotactic cytokines that regulate the trafficking and positioning of cells by activating the seven-transmembrane spanning $\mathrm{G}$ protein-coupled chemokine receptors (GPCR) or non G protein-coupled seven-transmembrane spanning receptors called atypical chemokine receptors (ACKR). Chemokines are basic proteins that also bind to glycosaminoglycans which play important roles in their biology. Chemokines are divided into four subfamilies based on the position of the first two N-terminal cysteine residues, including the CC, CXC, CX3C and XC subfamilies. Nearly 50 chemokines and 20 signaling chemokine receptors and 4 AKCRs have been identified. Dysregulated expression of chemokines and their corresponding receptors is implicated in many diseases, such as autoimmune and inflammatory diseases and cancer. Chemokines are essential coordinators of cellular migration and cell-cell interactions and, therefore, have great impact on tumor development. In the tumor microenvironment, tumor-associated host cells and cancer cells release an array of different chemokines, resulting in the recruitment and activation of different cell types that mediate the balance between antitumor and pro-tumor responses. In addition to their primary role as chemoattractants, chemokines are also involved in other tumor-related processes, including tumor cell growth, angiogenesis and metastasis. Therefore, further studies of the distinctions between the pro-tumor and antitumor activities of chemokines are warranted in order to develop more effective therapies against cancer. 